\title{
No diurnal variation of classical and candidate biomarkers of Alzheimer's disease in CSF
}

\author{
Claudia Cicognola ${ }^{1,2}$, Davide Chiasserini ${ }^{3}$, Paolo Eusebi ${ }^{3}$, Ulf Andreasson $^{1,2}$, Hugo Vanderstichele $^{4}$, \\ Henrik Zetterberg ${ }^{1,2,5}$, Lucilla Parnetti ${ }^{3^{*}+}$ and Kaj Blennow ${ }^{1,2+}$
}

\begin{abstract}
Background: Cerebrospinal fluid (CSF) biomarkers have gained increasing importance in the diagnostic work-up of Alzheimer's disease (AD). The core CSF biomarkers related to AD pathology (A $\beta 42, t$-tau and $p$-tau) are currently used in CSF diagnostics, while candidate markers of amyloid metabolism (A $338, A \beta 40$, sAPPa, sAPP $\beta$ ), synaptic loss (neurogranin), neuroinflammation (YKL-40), neuronal damage (VILIP-1) and genetic risk (apolipoprotein E) are undergoing evaluation. Diurnal fluctuation in the concentration of CSF biomarkers has been reported and may represent a preanalytical confounding factor in the laboratory diagnosis of AD. The aim of the present study was to investigate the diurnal variability of classical and candidate CSF biomarkers in a cohort of neurosurgical patients carrying a CSF drainage.
\end{abstract}

Method: Samples were collected from a cohort of 13 neurosurgical patients from either ventricular $(n=6)$ or lumbar $(n=7)$ CSF drainage at six time points during the day, 1-7 days following the neurosurgical intervention. Concentrations of the core biomarkers were determined by immunoassays.

Results: Although absolute values largely varied among subjects, none of the biomarkers showed significant diurnal variation. Site of drainage (lumbar vs. ventricular) did not influence this result. The different immunoassays used for tau and $A \beta$ markers provided similar results.

Conclusion: Time of day at CSF collection does not ultimately affect the concentration levels of classical and candidate AD biomarkers. Similar trends were found when using different immunoassays, thus corroborating the consistency of the data.

\section{Background}

Alzheimer's disease (AD) represents the most common neurodegenerative disease leading to dementia, and its prevalence is increasing and becoming a major health and socioeconomic issue [1]. AD pathology affects the brain several years before the clinical onset, which is characterized by a long asymptomatic phase followed by a prodromal phase with disturbances in episodic memory, ultimately leading to overt dementia [2]. To enable early initiation of treatment, especially the day disease-

\footnotetext{
* Correspondence: lucilla.parnetti@unipg.it

${ }^{\dagger}$ Equal contributors

${ }^{3}$ Section of Neurology, Department of Medicine, Center for Memory Disturbances, University of Perugia, Sant' Andrea delle Fratte, 06132 Perugia, Italy

Full list of author information is available at the end of the article
}

modifying drugs reach the clinic, early diagnosis is recommended, which is based on combining clinical symptoms and neuropsychological testing with biomarkers (CSF, imaging) reflecting AD pathology [3]. With respect to classical CSF biomarkers (A $\beta 42$, total tau, phosphorylated tau), large evidence has been collected about their reliability in supporting the diagnosis of AD [4-6].

In order to introduce the use of biomarkers as part of the routine diagnostic assessment, standardization of the procedures is mandatory. Preanalytical factors are reported to be the cause of at least $40-60 \%$ of the total variability in biomarker measurements [7]. Some key issues were identified for sample collection and analysis, such as presence of a CSF gradient, blood contamination of the sample, fasting state of the patient, and other laboratory procedures regarding collection and storage of 
the samples (collection tubes, aliquot volume, centrifugation, length and temperature of storage, number of freeze/thaw cycles).

The major focus of our study is the influence of time of day at CSF collection which, in a clinical routine schedule, may influence the results obtained. If proved significant, the diurnal fluctuations would affect the reliability of the CSF analysis depending on time at withdrawal. Only few studies have addressed this issue, giving inconsistent results: Bateman et al. first observed a significant decreasing trend of $A \beta$ during the $24 \mathrm{~h}$ in young individuals [8], an observation disproved in subsequent studies in older populations [9-11], the ones more likely to undergo CSF analysis to assess the risk of memory impairment and $\mathrm{AD}$. There is also no consensus on the time intervals between the CSF withdrawals or the volume to be taken.

Regarding analytical factors, different methods and assays may also represent another source of variability [12], but no study has been done on the performance and reliability of different assays when considering diurnal variation as a confounding factor.

\section{Candidate biomarkers}

Along with core biomarkers of $\mathrm{AD}$, other proteins are currently studied as candidate biomarkers, with significant results in their role in CSF diagnostics.

Biomarkers of the amyloidogenic pathway are being taken into account in $\mathrm{AD}$ diagnosis, since $\mathrm{A} \beta 42$ is produced from cleavage of amyloid precursor protein (APP). APP can be cleaved either by $\alpha$ - or $\beta$-secretase, releasing sAPP $\alpha$ or $\operatorname{sAPP} \beta$, respectively. After $\beta$-secretase cleavage, the remaining $C$-terminal fragment is further processed by $\gamma$-secretase which releases $A \beta 42$ along with $A \beta 38$ and $A \beta 40$ fragments, whereas no amyloidogenic fragments are produced after $\alpha$-secretase cleavage followed by $\gamma$-secretase. Since sAPP $\alpha$ was found decreased and sAPP $\beta$ increased in AD subjects, $\alpha$ - and $\beta$ - pathway were considered mutually exclusive [13-16]; however, subsequent studies showed positively correlated concentrations of sAPP $\alpha$ and $\beta$ [17-19], challenging the hypothesis of an imbalance between the two isoforms. The several critical steps of amyloid metabolism can also cause $A \beta$ fluctuations during the day: Dobrowolska et al. found diurnal fluctuations in sAPP $\alpha$, sAPP $\beta, A \beta 40$, and $A \beta 42$, diminishing with increased age [20].

Regarding genetic risk factors for $\mathrm{AD}, \mathrm{ApoE}$ is probably the most important and acknowledged. The three different isoforms exert a different effect on $\mathrm{AD}$ predisposition: ApoE4 increases the risk of $\mathrm{AD}$, whereas ApoE2 is a protective factor, compared to the most common variant ApoE3. Quantifying ApoE isoforms, especially ApoE4, could be a useful biological correlate in the study of $\mathrm{AD}$ pathology, but CNS and peripheral ApoE isoform turnover rates differ substantially, probably because the ApoE metabolism pathways are different in the central nervous system (CNS) and the periphery, as observed in a study by Wildsmith et al. [21]. Time-dependent fluctuations were also observed [21, 22].

Also neurogranin (a calmodulin-binding postsynaptic protein, involved in synaptic signaling, plasticity, longterm potentiation and memory consolidation) is currently studied as a potential biomarker. Synaptic loss has been reported to occur very early in the natural history of $\mathrm{AD}$, therefore neurogranin can be a valuable biomarker of early, possible preclinical, stage of the disease [23]. Neurogranin concentrations are significantly increased in mild cognitive impairment (MCI) $[24,25]$ and can be a predictive factor of conversion to dementia.

Among inflammatory markers we find YKL-40 (also known as chitinase-3-like protein 1), a protein mainly expressed by astrocytes. Even if its physiological function remains unclear [26], it was found to be upregulated in the AD brain from the preclinical stages of the disease and may have a role in $A \beta$ deposition [27]. Besides, as a biomarker, it could also give additional information on the inflammatory status of the AD brain. However, studies on its diagnostic and prognostic value still give conflicting results $[26,28]$.

Neuronal damage markers include VILIP-1 (visinin-like protein 1), a neuronal calcium sensor protein involved in calcium-mediated neurotoxicity. Not only VILIP-1 is significantly increased in $\mathrm{AD}$ and could help differentiate $\mathrm{AD}$ from other dementia, but it may also have an influence on tau metabolism [29, 30]. Moreover, when combined with $A \beta 42$, it is also a good predictor of cognitive decline [31, 32].

The diurnal variability of the aforementioned biomarkers has been poorly investigated and no studies are available on neurogranin, YKL-40 and VILIP-1. Also, most studies only use one immunoassay type and do not provide a comparison between different methods.

\section{Aim}

In this study we wanted to measure classical (A $A 42, t-t a u$, $\mathrm{p}$-tau) and candidate $(\mathrm{A} \beta 38, \mathrm{~A} \beta 40$, sAPP $\alpha$, sAPP $\beta$, apolipoprotein $\mathrm{E}$, neurogranin, YKL-40 and VILIP-1) AD biomarkers in CSF collected at six time points (08:00; 12:00; 16:00; 20:00, 00:00, 08:00) during $24 \mathrm{~h}$ to assess these markers for diurnal variation.

\section{Methods}

\section{Participants}

We enrolled a total of 13 patients, 8 males $(61.5 \%)$ and 5 females (38.5\%), with an age span between 26 and 82 years (Tables 1 and 2). All patients carried a CSF drainage after neurosurgical intervention for tumors, traumas or hemorrhages $(n=9)$ or for monitoring CSF pressure $(n=4) .7$ patients had a lumbar drainage $(53.8 \%)$ and 6 patients had a ventricular drainage (46.2\%), with no 
Table 1 Age, clinical features of the patients and type of drainage

\begin{tabular}{llll}
\hline Patient & Age & Case of drainage placement & Type of drainage \\
\hline 1 & 48 & Third ventricle tumor & ventricular \\
2 & 73 & Left middle cerebral artery infarct & ventricular \\
3 & 81 & Subarachnoid hemorrhage & ventricular \\
4 & 72 & Intracranial hemorrhage & lumbar \\
5 & 67 & Cerebellar hemorrhage & ventricular \\
6 & 69 & Cerebellopontine angle tumor & lumbar \\
7 & 74 & Normal pressure hydrocephalus & lumbar \\
8 & 36 & Head trauma & ventricular \\
9 & 63 & Intracranial hemorrhage & ventricular \\
10 & 58 & Tetraventricular hydrocephalus & lumbar \\
11 & 77 & Normal pressure hydrocephalus & lumbar \\
12 & 82 & Normal pressure hydrocephalus & lumbar \\
13 & 26 & Pseudotumor cerebri & lumbar \\
\hline
\end{tabular}

filter (Codman\&Shurtleff, Inc, Raynham, MA, USA). CSF was collected according to a standard protocol following international guidelines [33]. The study was approved by the local Ethical Committee and informed written consent was signed by all patients enrolled. The work was carried out according to the Declaration of Helsinki.

\section{CSF collection}

According to our protocol, $4 \mathrm{~mL}$ CSF were collected at six time points: $8.00,12.00,16.00,20.00,00.00$ and 8.00 . Haemorrhagic CSF samples were excluded; if the drainage was put after a trauma, haemorrhage or tumor surgery CSF samples collected had to be either clear or slightly and stably xantochromic $(<1000 \mathrm{rbc} / \mu \mathrm{L})$. CSF was

Table 2 Demographics (mean \pm standard deviation or count with percentages)

\begin{tabular}{ll}
\hline Age & $63.5 \pm 17.3$ \\
Sex & Male: 8 (61.5\%) \\
& Female: $5(38.5 \%)$ \\
Type of drainage & Lumbar: $7(53.8 \%)$ \\
& Ventricular: 6 (46.2 \%) \\
Cause of drainage & Cerebellar hemorrhage: 1 (7.7 \%) \\
placement & Cerebellopontine angle tumor: 1 (7.7\%) \\
& Head trauma: 1 (7.7 \%) \\
& Intracranial hemorrhage: 2 (15.4 \%) \\
& Left middle cerebral artery infarct: 1 (7.7 \%) \\
& Normal pressure hydrocephalus: 3 (23.1 \%) \\
& Pseudotumor cerebri: 1 (7.7 \%) \\
& Subarachnoid hemorrhage: 1 (7.7 \%) \\
& Tetraventricular hydrocephalus: 1 (7.7 \%) \\
& Third ventricle tumor: 1 (7.7 \%) \\
\hline
\end{tabular}

collected in polypropylene (PP) tubes (Sarsted, code: 62.610.201) and all samples were centrifuged (2000 $\mathrm{g} \times$ $10 \mathrm{~min}$, room temperature) no longer than $15 \mathrm{~min}$ after collection, aliquoted in $0.5 \mathrm{ml}$ aliquots (Sarsted, code: 72.730.007) and frozen at $-80^{\circ} \mathrm{C}$ pending analysis (Table 3).

\section{Assays}

A 442 , $t$-tau and $p$-tau were determined with ELISA kit (Fujirebio) in the Clinical Neurochemistry Laboratory of the University of Perugia (Perugia, Italy), according to the manufacturer's protocol.

$\mathrm{A} \beta 38, \mathrm{~A} \beta$ 40, $\mathrm{A} \beta$ 42, sAPP $\alpha, \mathrm{sAPP} \beta$, neurogranin, VILIP-1 and YKL-40 were analyzed in the Clinical Neurochemistry Laboratory of The Sahlgrenska Academy, University of Gothenburg (Mölndal, Sweden).

MSD V-PLEX Plus kits were used for $A \beta 38, A \beta 40$, $A \beta 42$, sAPP $\alpha$ and sAPP $\beta$, according to the manufacturer's protocol. ELISA kits from R\&D and Biovendor were used for YKL-40 and VILIP-1, respectively, according to the manufacturer's protocol.

An in-house developed assay was used for neurogranin, according to previous reports [24]. Briefly, in-house antibody Ng7 diluted in PBS was used as coating, a rabbit anti-neurogranin antibody (ab23570, cat. no. 07425, Upstate, Lake Placid, NY, USA) as primary and a goat anti-rabbit sulfo-tag antibody (MSD) for detection. Full-length Ng 1-78 protein (ADx) was used as calibrator for the standard curve.

$A \beta 38, A \beta 40, A \beta 42$, $t$-tau, and $A p o E$ were also measured with Euroimmun kits at ADx NeuroSciences (Gent, Belgium).

Table 3 CSF collection procedures and sample processing

\begin{tabular}{|c|c|}
\hline \multirow[t]{3}{*}{ Subjects: } & 13 neurosurgical patients carrying a CSF drainage \\
\hline & $8 \mathrm{M}, 5 \mathrm{~F} ; 26$ to 82 y \\
\hline & $\begin{array}{l}\text { Admitted for tumor, trauma, hemorrhage }(n=9) \text {, } \\
\text { CSF pressure monitoring }(n=4)\end{array}$ \\
\hline \multirow[t]{3}{*}{ CSF collection: } & From lumbar $(n=7)$ or ventricular $(n=6)$ drainage \\
\hline & Volume: 4 mL in PP Sarsted tubes, code: 62.610 .201 \\
\hline & Time of day: $8.00,12.00,16.00,20.00,00.00,8.00$ \\
\hline \multirow[t]{2}{*}{ CSF processing: } & Centrifugation within $15 \mathrm{~min}, 2000 \mathrm{~g} \times 10 \mathrm{~min}$ at RT \\
\hline & $0.5 \mathrm{~mL}$ aliquots in PP Sarsted tubes, code: 72.730 .007 \\
\hline CSF storage: & $\begin{array}{l}-80^{\circ} \mathrm{C} \text { freezer, with controlled temperature, alarm } \\
\text { and CO2 backup system }\end{array}$ \\
\hline Thawing: & RT with gentle shaking \\
\hline \multirow{6}{*}{$\begin{array}{l}\text { Biomarker } \\
\text { measurement: }\end{array}$} & Immunoassay (Fujirebio): A $11-42$, t-tau, p-tau181 \\
\hline & Immunoassay (Biovendor): VILIP-1 \\
\hline & Immunoassay (R\&D): YKL-40 \\
\hline & $\begin{array}{l}\text { Immunoassay (Euroimmun): A } \beta 1-38, A \beta 1-40, A \beta 1-42 \text {, } \\
\text { t-tau, ApoE }\end{array}$ \\
\hline & Immunoassay (in-house): neurogranin \\
\hline & Immunoassay (MSD): $A \beta x-38, A \beta x-40, A \beta x-42$, sAPPa, sAPP $\beta$ \\
\hline
\end{tabular}


Together with the quality control (QC) samples included in the kits, CSF pools were run in each plate as further internal controls to check for variability, as previously described [34]. Performance data (inter- and intra assay variability, recovery and linearity) can be found at the following references $[34,35]$.

\section{Statistical analysis}

Statistical analysis was performed using R v 13.1 and Graph Pad v 6.0. Continuous variables were reported as means, standard deviations and ranges. Categorical variables were shown as counts and percentages. Continuous variables were tested for normality with Shapiro-Wilk test. Differences in biomarkers levels across time points were tested by means of non-parametric version of repeated measurement ANOVA based on aligned ranks, due to the non-normality of the majority of parameters. Spearman's $r$ correlation coefficients of biomarkers concentrations were calculated pooling all the time points. Drainage type was tested as potential confounder in the fluctuations of biomarkers across time points. BlandAltman plots were drawn to investigate the agreement of different assays for some of the investigated biomarkers (A $338, \mathrm{~A} \beta 40, \mathrm{~A} \beta 42, \mathrm{t}$-tau). $P<0.05$ was chosen as the minimum level of statistical significance.

\section{Results}

Circadian trends in the individual classical and candidate biomarkers

$A \beta 38, A \beta 40, A \beta 42$ and $t$-tau measured with different methods, i.e. immunoassay by Euroimmun, Fujirebio, and MSD, showed significant positive correlations (Fig. 1). Results from Euroimmun vs. MSD highly correlated for both A $338(r=0.89, p<0.01,95 \%$ confidence interval $(\mathrm{CI})=$ 0.82 to 0.94$)$ and $\mathrm{A} \beta 40(r=0.87, p<0.01,95 \% \mathrm{CI}=0.74$ to 0.9 ). Similar results were found for $A \beta 42$ when comparing Euroimmun vs. MSD $(r=0.92, p<0.01,95 \% \mathrm{CI}=0.82$ to 0.93$)$, Euroimmun vs. Fujirebio $(r=0.81, p<0.01,95 \%$ $\mathrm{CI}=0.74$ to 0.9$)$ and MSD vs. Fujirebio $(r=0.86, p<0.01$, $95 \% \mathrm{CI}=0.74$ to 0.89 ) (Fig. 1 ).

There was also a positive correlation for tau when measured with Fujirebio and Euroimmun $(r=0.67$, $p<0.01,95 \% \mathrm{CI}=0.48$ to 0.8 ). Bland-Altman analysis was used to assess the agreement among the

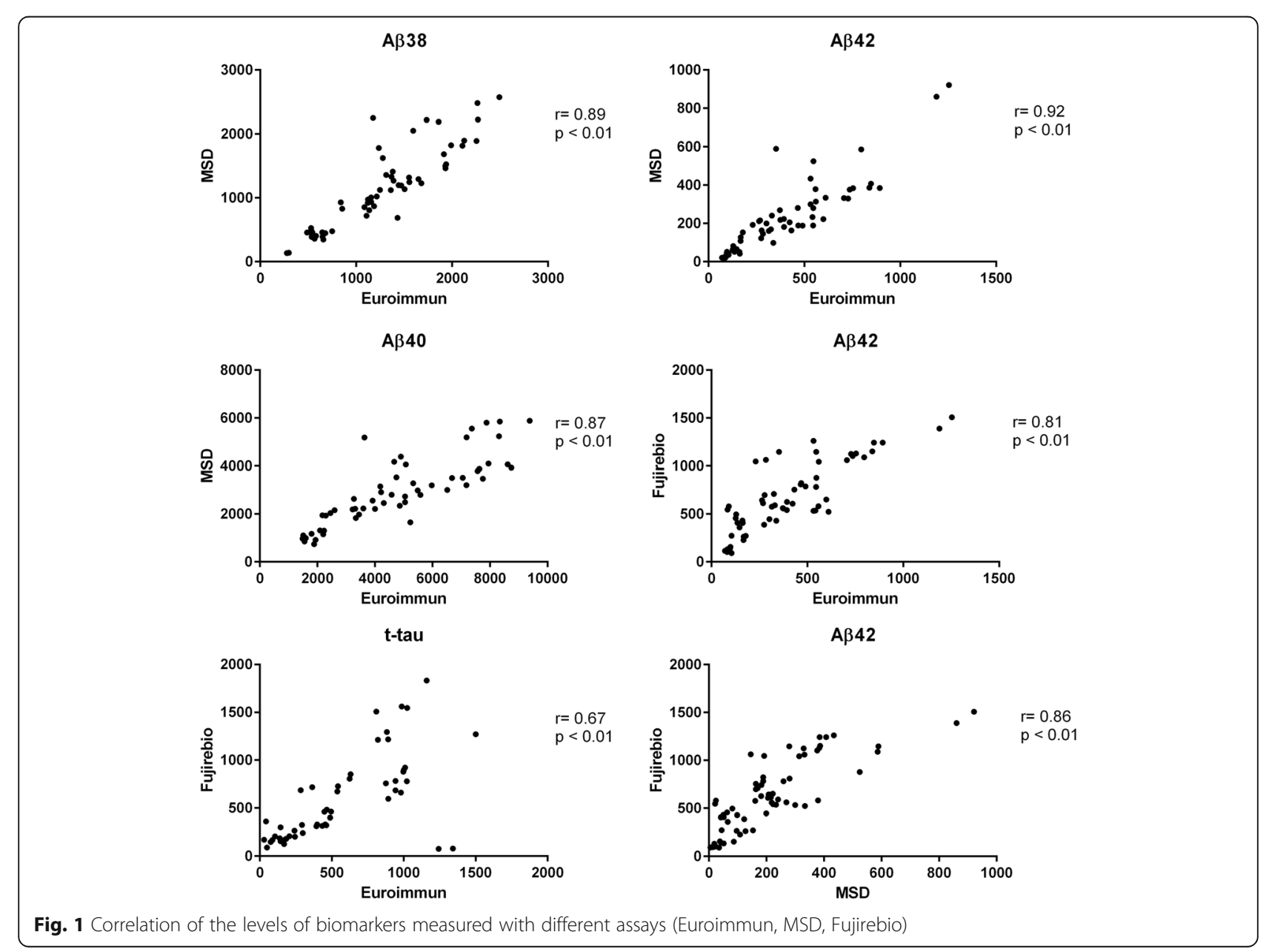


different assays for measuring absolute concentrations of the biomarkers (Additional file 1).

Notably, MSD assays overall showed differences either when compared to Euroimmun or Fujirebio assays. This was especially evident for $A \beta 42$ and $A \beta 40$ which showed the largest variation in the difference between the two assays across the whole range of concentrations (Additional file 1).

As expected from the different admittance diagnosis, sex and age of the population (Tables 1 and 2), absolute concentrations of the biomarkers were very different among patients (Table 4). For what concerns classical biomarkers, in the whole population $\mathrm{A} \beta 42$ ranged from 71 to $1253.4 \mathrm{pg} / \mathrm{mL}$, t-tau from 29 to $1501 \mathrm{pg} / \mathrm{mL}$ and p-tau from 15 to $113 \mathrm{pg} / \mathrm{mL}$.

Possibly due to their common metabolism pathway, amyloid metabolism markers (A $\beta 38, \mathrm{~A} \beta 40, \mathrm{~A} \beta 42, \mathrm{sAPP} \alpha$, sAPP $\beta$ ) showed similar trends over time in the whole population (Figs. 2 and 3). However, none of them had significant variation across time points $(p>0.05) . \operatorname{sAPP} \alpha, \operatorname{sAPP} \beta$ and $\beta$-amyloid fragments correlated positively with each other, with $\mathrm{r}$ values ranging from to 0.54 to $0.97(p>0.01)$ (Additional file 2). When measured with Euroimmun kits, $\mathrm{A} \beta 42 / \beta 40$ and $A \beta 38 / 40$ ratio showed no significant oscillations $(p>0.05)$. No significant intraindividual fluctuations over time were found for any of the $A \beta$ markers when measured with the different assays (for $A \beta 42$, see Additional file 3).

Neither T-tau nor P-tau did show a significant diurnal variation (Figs. 2 and 3). No significant oscillations were observed for Neurogranin, ApoE, YKL-40 and VILIP-1 $(p>0.05)$ (Fig. 2). Single patients showed some

Table 4 Concentration range of the biomarkers (with median)

\begin{tabular}{lll}
\hline Biomarker & Median (IQR) & Min - Max \\
\hline $\mathrm{A} \beta_{38}$ MSD $(\mathrm{pg} / \mathrm{mL})$ & $990.9(440.1-1396.0)$ & $33.3-2573.0$ \\
$\mathrm{~A} \beta_{40}$ MSD $(\mathrm{pg} / \mathrm{mL})$ & $2514.9(1154.8-3356.5)$ & $79.3-5880.5$ \\
$\mathrm{~A} \beta_{42}$ MSD $(\mathrm{pg} / \mathrm{mL})$ & $182.1(54.1-266.6)$ & $5.5-921.2$ \\
$\mathrm{~A} \beta_{38}$ Euroimmun $(\mathrm{pg} / \mathrm{mL})$ & $1247.7(1120.9-1462.0)$ & $277.5-2492.5$ \\
$\mathrm{~A} \beta_{40}$ Euroimmun $(\mathrm{pg} / \mathrm{mL})$ & $4669.0(3605.0-5305.0)$ & $1487.0-9382.2$ \\
$\mathrm{~A} \beta_{42}$ Euroimmun $(\mathrm{pg} / \mathrm{mL})$ & $337.9(191.9-521.8)$ & $70.6-1253.4$ \\
t-tau Euroimmun $(\mathrm{pg} / \mathrm{mL})$ & $464.7(223.0-917.6)$ & $29.3-1501.0$ \\
A $\beta_{42}$ Fujirebio $(\mathrm{pg} / \mathrm{mL})$ & $568.0(294.5-786.0)$ & $91.0-1507.0$ \\
t-tau Fujirebio $(\mathrm{pg} / \mathrm{mL})$ & $319.0(180.2-742.2)$ & $72.0-1832.0$ \\
p-tau (pg/mL) & $43.0(30.3-52.8)$ & $15.0-113.0$ \\
sAPPa (ng/mL) & $60.4(32.5-178.8)$ & $4.3-429.6$ \\
sAPP $\beta$ & $66.4(25.5-175.0)$ & $1.4-313.6$ \\
YKL-40 (ng/mL) & $264.6(151.9-348.4)$ & $65.4-1020.0$ \\
VILIP-1 (pg/mL) & $113.5(99.0-135.0)$ & $72.0-781.0$ \\
Neurogranin (pg/mL) & $354.5(275.9-435.4)$ & $46.3-1291.1$ \\
ApoE (ng/mL) & $24.5(18.8-44.4)$ & $3.4-104.2$ \\
\hline
\end{tabular}

variations for specific proteins but globally no significant fluctuations over time were observed.

\section{Site of drainage}

When site of drainage was considered as a covariate in non-parametric repeated measurements ANOVA based on aligned ranks, variability in some of the proteins $(\mathrm{A} \beta 38$, A 340 , YKL40, $t$-tau) was found to be significantly explained by the site of drainage $(p<0.05)$, with generally higher concentration in CSF from ventricular catheter. Anyway, subgroup analyses restricted to lumbar or ventricular catheter showed no significant changes across the time points analysed ( $p>0.05)$ (Fig. 4). Similar results were obtained when the analysis was repeated considering only patients with normal pressure hydrocephalus $(n=3, p>0.05)$.

\section{Discussion}

In our study we assessed the possible circadian oscillation of classical (A $\beta 42, \mathrm{t}$-tau, $\mathrm{p}$-tau) and candidate $(\mathrm{A} \beta 38, \mathrm{~A} \beta 40, \mathrm{sAPP} \alpha, \mathrm{sAPP} \beta$, apolipoprotein $\mathrm{E}$, neurogranin, YKL-40, VILIP-1) CSF AD biomarkers. Previous studies addressed the issue of diurnal fluctuations of $A \beta$ and tau in CSF (for review see [36]), however, a significant circadian oscillation of $\mathrm{A} \beta$ was detected only in the study by Bateman and colleagues [8]. Moreover, the study included subjects significantly younger than the ones who usually undergo lumbar puncture as a diagnostic procedure (23 to 78 years old).

Subsequent studies did not observe the same trend [9-11]. Bjerke et al. led a study in healthy subjects undergoing knee surgery and observed a slight decrease in A 342 levels, which tended to return to baseline after $24 \mathrm{~h}$ [10]. Slats et al. compared the trends of $A \beta$ in healthy and mild AD subjects, noticing no significant diurnal variation and also a less pronounced circadian pattern compared with the one in younger subjects (59 to 85 years old) [11]. Only Moghekar et al. examined also tau along with $\mathrm{A} \beta$, in a cohort of normal pressure hydrocephalus subjects, but no diurnal fluctuation of the biomarkers was reported [9].

Our study is in line with the majority of the results from previous ones, but we also investigated the possible oscillations of the latest candidate $\mathrm{AD}$ biomarkers (neurogranin, YKL-40, VILIP-1). We collected a smaller volume of CSF $(4 \mathrm{~mL}$ ) than in previous studies (from 6 to $40 \mathrm{~mL}$ ) to avoid possible fluctuations due to tapping large volumes of CSF, and we compared results from ventricular CSF and lumbar CSF to detect a possible gradient effect.

Although CSF protein concentrations were significantly different from one patient to another, none of the biomarkers showed a significant diurnal variation. The ranges of the biomarkers were very different from the ones available from the literature on optimal cut-offs for 


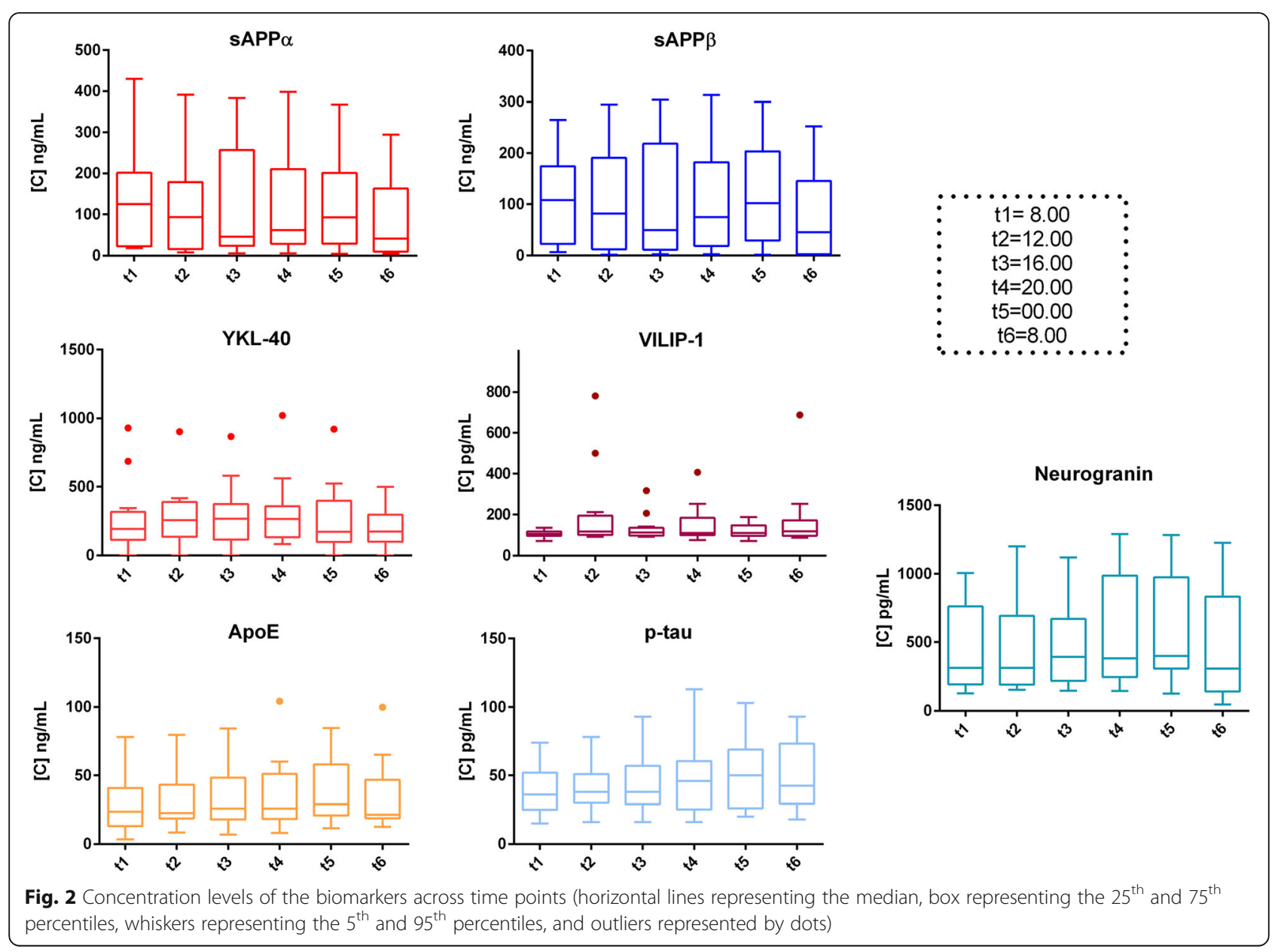

AD biomarkers [37]. This is mostly due to the heterogeneity of the population, different for sex, age and diagnosis at admission (Tables 1 and 2). The subjects did all undergo an invasive procedure for catheter insertion, which might have affected the levels of the biomarkers in CSF. Moreover, 9 out of 13 subjects underwent brain surgery for tumours, haemorrhage or head trauma prior to drainage placement, which is most likely the reason for the generally upregulated values of inflammatory and brain injury markers (tau, YKL-40) in the population. Not to be excluded is also the presence of a possible underlying $\mathrm{AD}$ pathology in the older subjects. It is remarkable, however, that even if set on different ranges between subjects, the overall levels of the individual biomarkers are very stable over time and do not seem to be affected by external factors.

Site of drainage significantly influenced the variability of some of the biomarkers (A $\beta 38, A \beta 40$, YKL40, t-tau), as previously described [38], with generally higher concentration in CSF from ventricular catheter, most likely due to the increased concentrations of injury markers in the site of the surgical intervention or brain damage in patients carrying a ventricular drainage.
Some of the analytes tested in this work were measured with different immunoassays (Fujirebio, MSD, and Euroimmun). Absolute concentrations of the biomarkers using different immunoassays varied widely, possibly due to the different sources for the calibrators used by the manufacturers, as well as to the different antibodies used for coating and detection. No previous studies on circadian rhythms of CSF biomarkers have been performed comparing these three different techniques, which nevertheless showed good inter-assay correlations, proving the reliability of the methods in use and supporting the lack of circadian oscillations independently from the technique.

The main limitation of this study was the population, mostly admitted to hospital for traumatic intervention, limited in number and very different for age and disease. However, patients with normal pressure hydrocephalus, which were admitted to hospital only for diagnostic purposes and represented a more selected population, did not show significant diurnal variability as well. Obviously, to find ventricular CSF samples suitable for biomarker analysis is not easily manageable, due to the severe underlying conditions that lead to the insertion of a ventricular 

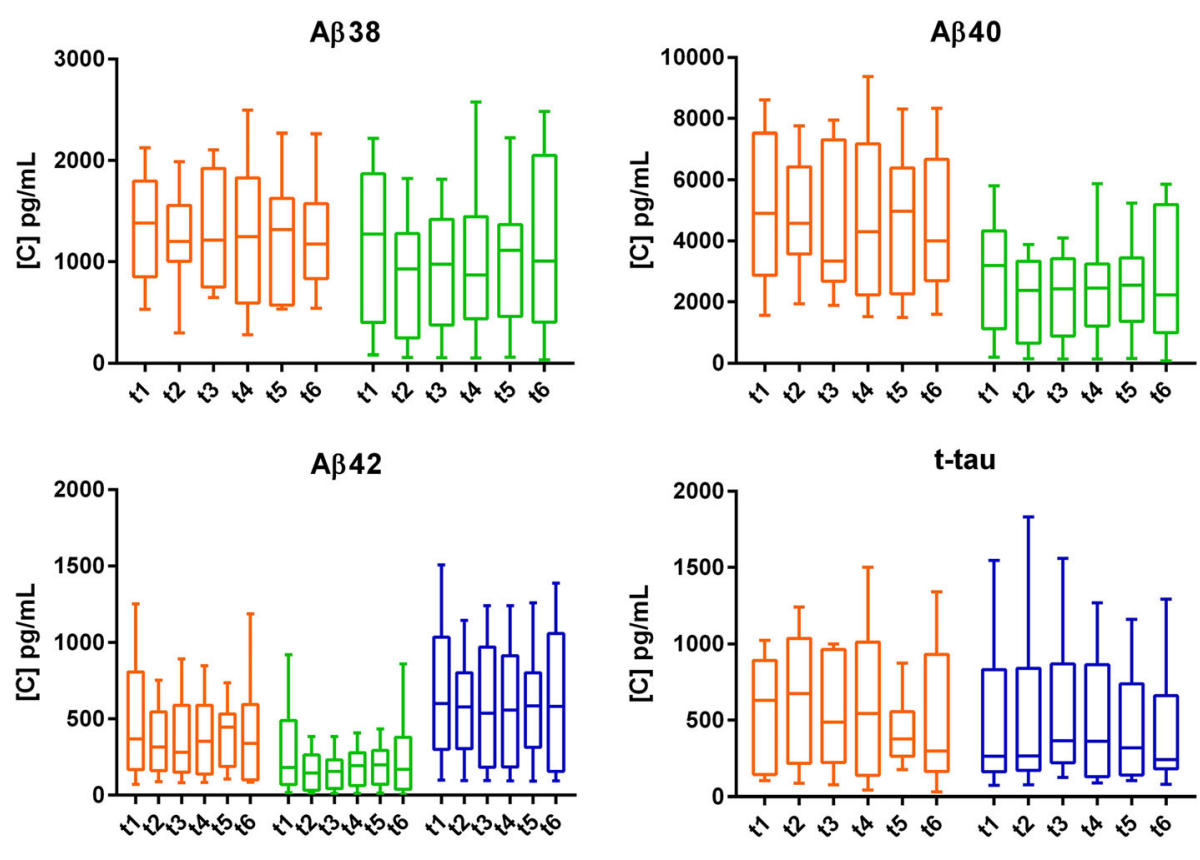

$\square$ Euroimmun

MSD

Fujirebio

Fig. 3 Concentration levels of several biomarkers measured with different assays (Euroimmun, MSD, Fujirebio) across time points (horizontal lines representing the median, box representing the $25^{\text {th }}$ and $75^{\text {th }}$ percentiles, whiskers representing the $5^{\text {th }}$ and $95^{\text {th }}$ percentiles)
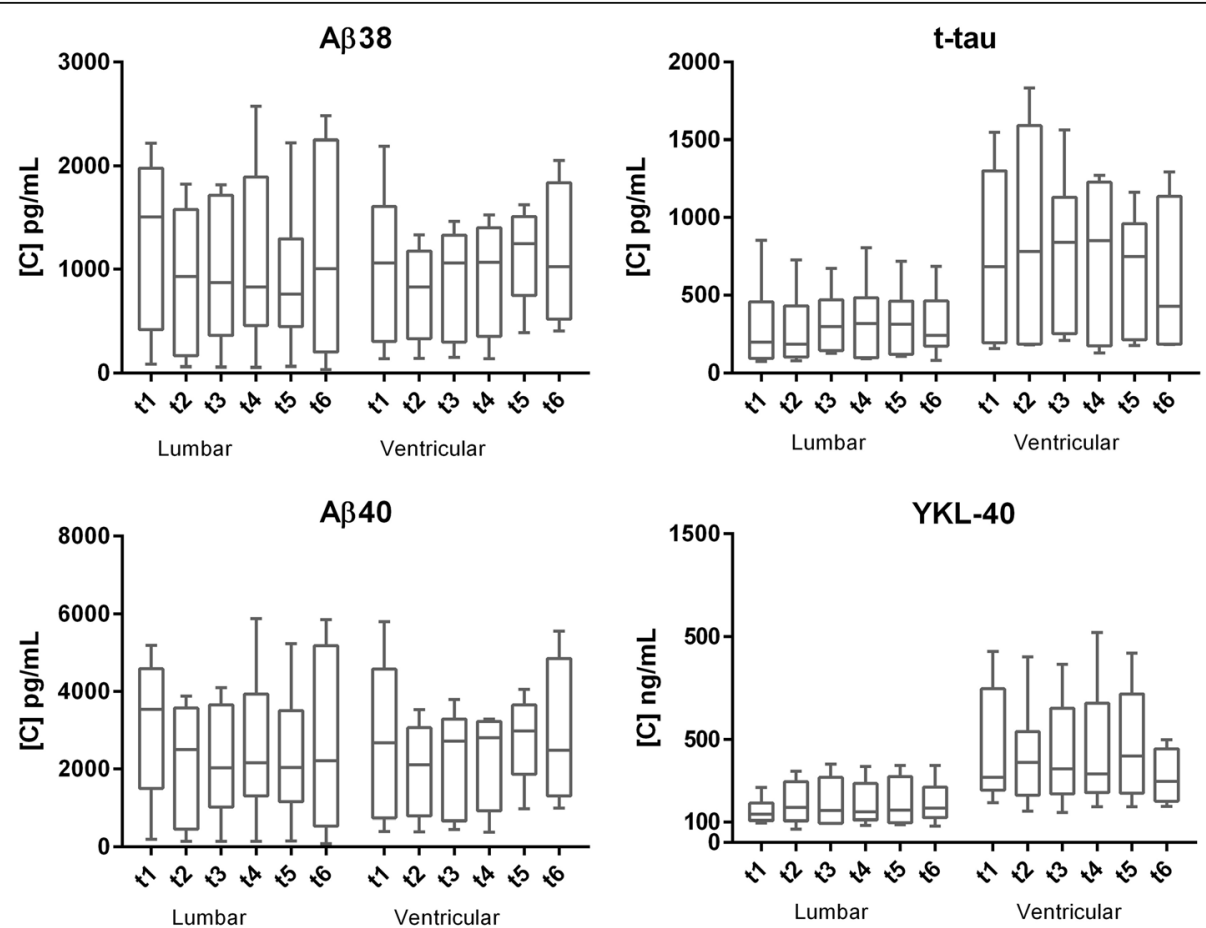

Fig. 4 Concentration levels of several biomarkers with different type of drainage (lumbar or ventricular) across time points (horizontal lines representing the median, box representing the $25^{\text {th }}$ and $75^{\text {th }}$ percentiles, whiskers representing the $5^{\text {th }}$ and $95^{\text {th }}$ percentiles). Pictures not showed for non-significant comparisons 
catheter. Same is for lumbar CSF: not being possible to perform multiple lumbar punctures during the day, repeated measurements at different time points can only be done after insertion of a drainage, which is seen as a dangerous procedure by most of the population, making it problematic to enrol healthy controls and AD subjects in larger studies. Further efforts are needed to raise public awareness about the minor risks of this kind of procedure, especially in view of an early diagnosis of AD pathology.

\section{Conclusions}

In view of standardizing CSF collection and analysis procedures, the absence of an influence on classical and candidate CSF biomarker of time at sampling make them a valuable asset in the diagnostic work-up of AD. Independence from time of day is also crucial for clinical practitioners: being bound to fixed hospital schedules, the possibility to perform the lumbar puncture at any time of the day is of great importance.

An increasing number of tools are already being developed for the measurement of these analytes in CSF and preliminary results show robustness and significant overlap between different methods, proving their reliability and reproducibility for biomarker analysis. However, larger and selected populations are needed to increase the power of these findings.

\section{Additional files}

Additional file 1: Bland-Altman plot for investigating the agreement between assays (mean difference and $95 \%$ limits of agreement). (JPG 2632 kb)

Additional file 2: Scatterplot and Spearman correlation coefficients with p-values for amyloid metabolism biomarkers measured with the three different assays. (JPG 1903 kb)

Additional file 3: Intraindividual concentration levels of A 42 across time points measured with different assays (Euroimmun, MSD, Fujirebio). (JPG $2223 \mathrm{~kb}$ )

Additional file 4: Raw data. (XLSX $28 \mathrm{~kb})$

\section{Acknowledgments}

The authors would like to thank Cristiano Spaccatini, Ulrika Törnqvist and Leentje Demeyer for their technical assistance and Professor Paolo Lunardi for allowing access to the Section of Neurosurgery (Santa Maria della Misericordia Hospital, University of Perugia, Italy).

\section{Funding}

Blennow received funding from Research Council, Sweden; the Swedish Brain Foundation; the Swedish Alzheimer Foundation; and Torsten Söderberg Foundation, Sweden. Parnetti received funding from Michael J. Fox Foundation for Parkinson's Disease.

\section{Availability of data and materials}

The dataset supporting the conclusions of this article is included within the article and its additional files (Additional file 4).

\section{Authors' contributions}

CC collected the samples, performed the experiments, analyzed the data and wrote the manuscript. DC performed the experiments, designed and supervised the analysis and participated in the drafting of the manuscript. PE performed the statistical analysis and participated in the drafting of the manuscript. UA coordinated the analysis at the Clinical Neurochemistry Laboratory of The Sahlgrenska Academy, University of Gothenburg (Mölndal, Sweden) and reviewed the manuscript. HV run the analysis at ADx NeuroSciences (Gent, Belgium) and reviewed the manuscript. $\mathrm{HZ}$ participated in the design of the study and reviewed the manuscript. LP and KB designed the study, gave financial support and reviewed the manuscript. All authors read and approved the final manuscript.

\section{Authors' information}

Not applicable.

\section{Competing interests}

Blennow has served at advisory boards and as a consultant for IBL International, Fujirebio Europe, and Roche Diagnostics. Parnetti obtained research reagents from Fujirebio and Euroimmun AG. Vanderstichele is co-founder of ADx NeuroSciences and founder of Biomarkable bvba. The other authors report no disclosures for this paper.

\section{Consent for publication}

Not applicable.

\section{Ethics approval and consent to participate}

The study was approved by the local Ethical Committee (Comitato Etico delle Aziende Sanitarie della Regione Umbria - CEAS Umbria) and informed written consent was signed by all patients enrolled. The work was carried out according to the Declaration of Helsinki.

\section{Author details}

${ }^{1}$ Institute of Neuroscience and Physiology, Department of Psychiatry and Neurochemistry, The Sahlgrenska Academy at University of Gothenburg, House V3, SU / Mölndal hospital, Göteborgsvägen 31, SE-431 80 Mölndal, Sweden. ${ }^{2}$ Clinical Neurochemistry Laboratory, Sahlgrenska University Hospital, SE-431 80 Mölndal, Sweden. ${ }^{3}$ Section of Neurology, Department of Medicine, Center for Memory Disturbances, University of Perugia, Sant' Andrea delle Fratte, 06132 Perugia, Italy. ${ }^{4} \mathrm{ADx}$ NeuroSciences, Gent, Belgium. ${ }^{5} \mathrm{UCL}$ Institute of Neurology, Queen Square, London, UK.

Received: 28 May 2016 Accepted: 27 August 2016

Published online: 07 September 2016

\section{References}

1. Prince M, Bryce R, Albanese E, Wimo A, Ribeiro W, Ferri CP. The global prevalence of dementia: a systematic review and metaanalysis. Alzheimers Dement. 2013;9(1):63-75. e62.

2. Sperling RA, Aisen PS, Beckett LA, Bennett DA, Craft S, Fagan AM, Iwatsubo T, Jack Jr CR, Kaye J, Montine TJ, et al. Toward defining the preclinical stages of Alzheimer's disease: recommendations from the National Institute on Aging-Alzheimer's Association workgroups on diagnostic guidelines for Alzheimer's disease. Alzheimers Dement. 2011;7(3):280-92.

3. Dubois B, Feldman HH, Jacova C, Hampel H, Molinuevo JL, Blennow K, DeKosky ST, Gauthier S, Selkoe D, Bateman R, et al. Advancing research diagnostic criteria for Alzheimer's disease: the IWG-2 criteria. The Lancet Neurology. 2014;13(6):614-29.

4. Blennow K, Zetterberg $H$. The past and the future of Alzheimer's disease CSF biomarkers-a journey toward validated biochemical tests covering the whole spectrum of molecular events. Frontiers in neuroscience. 2015;9:345

5. Rosen C, Hansson O, Blennow K, Zetterberg H. Fluid biomarkers in Alzheimer's disease - current concepts. Mol Neurodegener. 2013;8:20.

6. Molinuevo JL, Blennow K, Dubois B, Engelborghs S, Lewczuk P, PerretLiaudet A, Teunissen CE, Parnetti L. The clinical use of cerebrospinal fluid biomarker testing for Alzheimer's disease diagnosis: a consensus paper from the Alzheimer's Biomarkers Standardization Initiative. Alzheimers Dement. 2014;10(6):808-17.

7. Vanderstichele H, Bibl M, Engelborghs S, Le Bastard N, Lewczuk P, Molinuevo $J$, Parnetti L, Perret-Liaudet A, Shaw LM, Teunissen C, et al. Standardization of preanalytical aspects of cerebrospinal fluid biomarker testing for Alzheimer's disease diagnosis: a consensus paper from the Alzheimer's Biomarkers Standardization Initiative. Alzheimers Dement. 2012;8(1):65-73.

8. Bateman RJ, Wen G, Morris JC, Holtzman DM. Fluctuations of CSF amyloidbeta levels: implications for a diagnostic and therapeutic biomarker. Neurology. 2007;68(9):666-9. 
9. Moghekar A, Goh J, Li M, Albert M, O'Brien RJ. Cerebrospinal fluid Abeta and tau level fluctuation in an older clinical cohort. Arch Neurol. 2012;69(2):246-50

10. Bjerke M, Portelius E, Minthon L, Wallin A, Anckarsater H, Anckarsater R, Andreasen N, Zetterberg H, Andreasson U, Blennow K: Confounding factors influencing amyloid Beta concentration in cerebrospinal fluid. Int J Alzheimers Dis. 2010;2010. doi: 10.4061/2010/986310

11. Slats D, Claassen JA, Spies PE, Borm G, Besse KT, van Aalst W, Tseng J, Sjogren MJ, Olde Rikkert MG, Verbeek MM. Hourly variability of cerebrospinal fluid biomarkers in Alzheimer's disease subjects and healthy older volunteers. Neurobiol Aging. 2012;33(4):831. e831-839.

12. Mattsson N, Andreasson U, Persson S, Carrillo MC, Collins S, Chalbot S, Cutler N, Dufour-Rainfray D, Fagan AM, Heegaard NH, et al. CSF biomarker variability in the Alzheimer's Association quality control program. Alzheimers Dement. 2013;9(3):251-61.

13. Olsson A, Hoglund $K$, Sjogren M, Andreasen N, Minthon L, Lannfelt L, Buerger K, Moller HJ, Hampel H, Davidsson P, et al. Measurement of alphaand beta-secretase cleaved amyloid precursor protein in cerebrospinal fluid from Alzheimer patients. Exp Neurol. 2003;183(1):74-80.

14. Sennvik K, Fastbom J, Blomberg M, Wahlund LO, Winblad B, Benedikz E. Levels of alpha- and beta-secretase cleaved amyloid precursor protein in the cerebrospinal fluid of Alzheimer's disease patients. Neurosci Lett. 2000;278(3):169-72.

15. Lannfelt L, Basun H, Wahlund LO, Rowe BA, Wagner SL. Decreased alphasecretase-cleaved amyloid precursor protein as a diagnostic marker for Alzheimer's disease. Nature medicine. 1995;1(8):829-32

16. Vassar R. beta-Secretase, APP and Abeta in Alzheimer's disease. Sub-cellular biochemistry. 2005;38:79-103.

17. Alexopoulos P, Tsolakidou A, Roselli F, Arnold A, Grimmer T, Westerteicher C, Leante MR, Forstl H, Livrea P, Kurz A, et al. Clinical and neurobiological correlates of soluble amyloid precursor proteins in the cerebrospinal fluid. Alzheimers Dement. 2012;8(4):304-11.

18. Gabelle A, Roche S, Geny C, Bennys K, Labauge P, Tholance Y, Quadrio I, Tiers L, Gor B, Chaulet C, et al. Correlations between soluble alpha/beta forms of amyloid precursor protein and Abeta38, 40, and 42 in human cerebrospinal fluid. Brain Res. 2010;1357:175-83.

19. Lewczuk P, Kamrowski-Kruck H, Peters O, Heuser I, Jessen F, Popp J, Burger K, Hampel H, Frolich L, Wolf S, et al. Soluble amyloid precursor proteins in the cerebrospinal fluid as novel potential biomarkers of Alzheimer's disease: a multicenter study. Mol Psychiatry. 2010;15(2):138-45.

20. Dobrowolska JA, Kasten T, Huang Y, Benzinger TL, Sigurdson W, Ovod V, Morris JC, Bateman RJ. Diurnal patterns of soluble amyloid precursor protein metabolites in the human central nervous system. PLoS One. 2014;9(3):e89998.

21. Wildsmith KR, Basak JM, Patterson BW, Pyatkivskyy Y, Kim J, Yarasheski KE, Wang JX, Mawuenyega KG, Jiang H, Parsadanian M, et al. In vivo human apolipoprotein E isoform fractional turnover rates in the CNS. PLoS One. 2012;7(6):e38013.

22. Yamauchi K, Tozuka M, Nakabayashi T, Sugano M, Hidaka H, Kondo Y, Katsuyama T. Apolipoprotein E in cerebrospinal fluid: relation to phenotype and plasma apolipoprotein E concentrations. Clin Chem. 1999;45(4):497-504.

23. Thorsell A, Bjerke M, Gobom J, Brunhage E, Vanmechelen E, Andreasen N, Hansson O, Minthon L, Zetterberg H, Blennow K. Neurogranin in cerebrospinal fluid as a marker of synaptic degeneration in Alzheimer's disease. Brain Res. 2010;1362:13-22.

24. Kvartsberg H, Duits FH, Ingelsson M, Andreasen N, Ohrfelt A, Andersson K, Brinkmalm G, Lannfelt L, Minthon L, Hansson O, et al. Cerebrospinal fluid levels of the synaptic protein neurogranin correlates with cognitive decline in prodromal Alzheimer's disease. Alzheimers Dement. 2015;11(10):1180-90.

25. De Vos A, Jacobs D, Struyfs H, Fransen E, Andersson K, Portelius E, Andreasson U, De Surgeloose D, Hernalsteen D, Sleegers K, et al. C-terminal neurogranin is increased in cerebrospinal fluid but unchanged in plasma in Alzheimer's disease. Alzheimers Dement. 2015;11:1461.

26. Craig-Schapiro R, Perrin RJ, Roe CM, Xiong C, Carter D, Cairns NJ, Mintun MA, Peskind ER, Li G, Galasko DR, et al. YKL-40: a novel prognostic fluid biomarker for preclinical Alzheimer's disease. Biol Psychiatry. 2010;68(10):903-12.

27. Rosen C, Andersson CH, Andreasson U, Molinuevo JL, Bjerke M, Rami L, Llado A, Blennow K, Zetterberg H. Increased levels of chitotriosidase and YKL-40 in cerebrospinal fluid from patients with Alzheimer's disease. Dement Geriatr Cogn Dis Extra. 2014;4(2):297-304.

28. Mattsson N, Tabatabaei S, Johansson P, Hansson O, Andreasson U, Mansson JE, Johansson JO, Olsson B, Wallin A, Svensson J, et al. Cerebrospinal fluid microglial markers in Alzheimer's disease: elevated chitotriosidase activity but lack of diagnostic utility. Neuromolecular Med. 2011;13(2):151-9.
29. Luo X, Hou L, Shi H, Zhong X, Zhang Y, Zheng D, Tan Y, Hu G, Mu N, Chan J, et al. CSF levels of the neuronal injury biomarker visinin-like protein-1 in Alzheimer's disease and dementia with Lewy bodies. J Neurochem. 2013;127(5):681-90.

30. Schnurra I, Bernstein HG, Riederer P, Braunewell KH. The neuronal calcium sensor protein VILIP-1 is associated with amyloid plaques and extracellular tangles in Alzheimer's disease and promotes cell death and tau phosphorylation in vitro: a link between calcium sensors and Alzheimer's disease? Neurobiol Dis. 2001;8(5):900-9.

31. Tarawneh R, D'Angelo G, Macy E, Xiong C, Carter D, Cairns NJ, Fagan AM, Head D, Mintun MA, Ladenson JH, et al. Visinin-like protein-1: diagnostic and prognostic biomarker in Alzheimer disease. Ann Neurol. 2011;70(2):274-85.

32. Tarawneh R, Lee JM, Ladenson JH, Morris JC, Holtzman DM. CSF VILIP-1 predicts rates of cognitive decline in early Alzheimer disease. Neurology. 2012:78(10):709-19.

33. Teunissen CE, Petzold A, Bennett JL, Berven FS, Brundin L, Comabella M, Franciotta D, Frederiksen $J$, Fleming JO, Furlan R, et al. A consensus protocol for the standardization of cerebrospinal fluid collection and biobanking. Neurology. 2009;73(22):1914-22.

34. Chiasserini D, Biscetti L, Farotti L, Eusebi P, Salvadori N, Lisetti V, Baschieri F, Chipi E, Frattini G, Stoops E, et al. Performance Evaluation of an Automated ELISA System for Alzheimer's Disease Detection in Clinical Routine. J Alzheimers Dis. 2016;54(1):55-67.

35. Sutphen $C L$, Jasielec MS, Shah AR, Macy EM, Xiong C, Vlassenko AG, Benzinger TL, Stoops EE, Vanderstichele HM, Brix B, et al. Longitudinal cerebrospinal fluid biomarker changes in preclinical Alzheimer disease during middle age. JAMA Neurol. 2015;72(9):1029-42.

36. Cicognola C, Chiasserini D, Parnetti L. Preanalytical confounding factors in the analysis of cerebrospinal fluid biomarkers for Alzheimer's disease: the issue of diurnal variation. Front Neurol. 2015;6:143.

37. Fourier A, Portelius E, Zetterberg H, Blennow K, Quadrio I, Perret-Liaudet A Pre-analytical and analytical factors influencing Alzheimer's disease cerebrospinal fluid biomarker variability. Clin Chim Acta. 2015;449:9-15.

38. Brandner S, Thaler C, Lelental N, Buchfelder M, Kleindienst A, Maler JM, Kornhuber J, Lewczuk P. Ventricular and lumbar cerebrospinal fluid concentrations of Alzheimer's disease biomarkers in patients with normal pressure hydrocephalus and posttraumatic hydrocephalus. J Alzheimers Dis. 2014;41(4):1057-62.

\section{Submit your next manuscript to BioMed Central and we will help you at every step:}

- We accept pre-submission inquiries

- Our selector tool helps you to find the most relevant journal

- We provide round the clock customer support

- Convenient online submission

- Thorough peer review

- Inclusion in PubMed and all major indexing services

- Maximum visibility for your research

Submit your manuscript at www.biomedcentral.com/submit
C Biomed Central 